\title{
Use of electroacoustic localisation in the management of intraocular foreign bodies
}

\author{
G. A. SUTTON \\ From the Birmingham and Midland Eye Hospital, Church Street, Birmingham B3 2NS
}

SUMMARY A new model of the electroacoustic discriminator and localiser has been put into production, and this paper reports on the preproduction model during its trial period in the management of patients with intraocular foreign bodies. Its particular advantage in localisation at the time of surgery and the procedure for using it are described. The visual results following surgical extraction of 17 cases of magnetic foreign bodies in the posterior segment are outlined.

The success of removing a magnetic foreign body depends on accurate localisation, so that the best surgical approach can be made in each case. We have found the transretinal route of extraction to be the most satisfactory and prefer it to the pars plana route in most cases, as it avoids drawing the foreign body through formed vitreous base. The long-term outcome is good in $80 \%$ of cases even if conventional lens surgery is necessary. A pars plana vitrectomy is not required in most cases. The anterior route of extraction has been found to be associated with a high incidence of complications and should not be attempted for posterior segment foreign bodies (Roper-Hall, 1954).

\section{Assessment}

The suspicion of a foreign body is aroused by the history and clinical examination, and its nature can usually be ascertained by questioning the patient. Careful clinical examination of the anterior segment may disclose an entry wound, the size of which is a useful indicator of the foreign body size. Similarly, examining the trajectory of the foreign body can be helpful in deciding into which part of the eye the foreign body is likely to have penetrated.

The early clinical assessment should include fundus examination by indirect ophthalmoscopy, which is generally possible if carried out within 24 hours of the injury even if there are advancing lens opacities. At this time a decision on the degree of vitreous haemorrhage present should be made. This finding will significantly influence the future management with respect to the need for vitrectomy. Indirect ophthalmoscopy often reveals the foreign body, but in some $15 \%$ to $20 \%$ of cases of posterior

Correspondence to Mr G. A. Sutton. segment foreign body the view is limited to haemorrhage.

Anteroposterior and lateral $x$-rays are essential whenever an intraocular foreign body is suspected. The preoperative clinical assessment is supported by radiological localisation by the limbal ring method, which we have found to be the most accurate. The relative inexperience of most individual radiographers in general radiological departments makes the occasional $x$-ray localisation less accurate; when the foreign body lies in the posterior segment of the globe, the amount of potential error is considerable. It must be borne in mind that the position of the patient at the time of $x$-ray localisation may be different from that of the operating table. In consequence a mobile intraocular foreign body may come to lie at some considerable distance from its $x$-ray localisation. For this reason both indirect ophthalmoscopy and the procedure of operative localisation with the electroacoustic apparatus described below are used to recheck the $x$-ray findings.

The bewildering array of radiological techniques of localisation which have been described is a measure of their limitations. The author has no personal experience of the image intensifier for localisation. Ultrasonography has proved to be of very limited application in localisation and is no longer routinely used in this hospital.

In doubtful cases the magnetic nature of the foreign body is confirmed before surgery by using the discriminator capability of the apparatus. This clearly avoids a futile surgical operation by an incorrectly chosen procedure.

\section{Electroacoustic discriminator and localiser}

The new electroacoustic discriminator and localiser (Fig. 1) is an improved version of the original 
Fig. 1 The electroacoustic discriminator and localiser

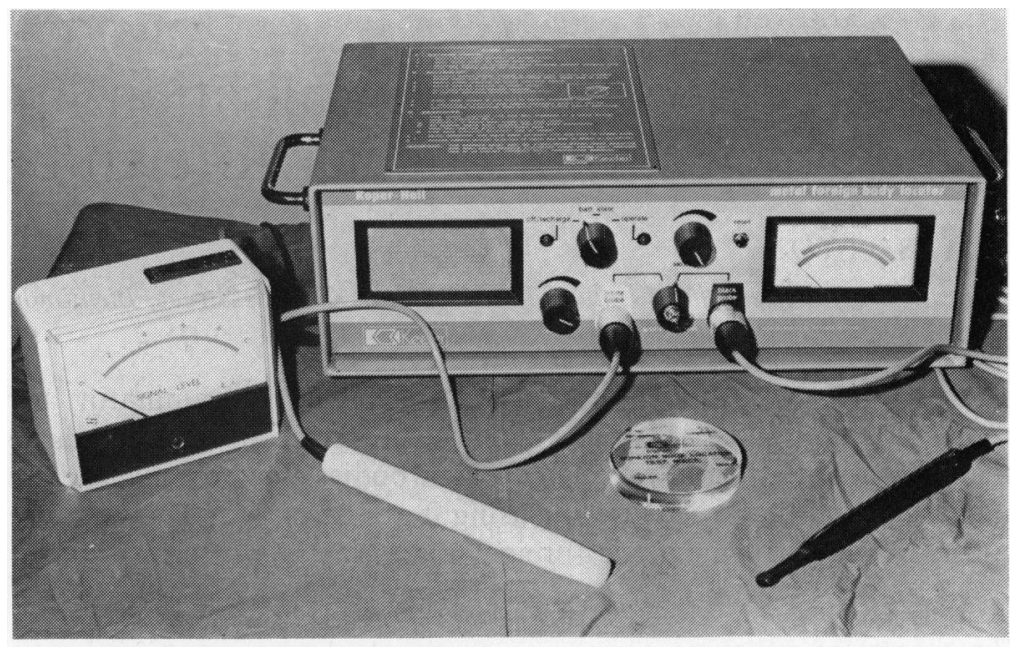

instrument as devised by Roper-Hall. The electrical principle of its construction is that of a small coil located in a searching probe which is balanced as part of an electrical bridge, the other half of which is remote from the probe head. A magnetic foreign body in the magnetic field of the searching coil causes the magnetic field to intensify and increases the inductance. In the case of a nonmagnetic foreign body the reverse occurs. These alterations in the inductance are recordable on the instrument console both visibly by a needle deflection of the meter and audibly through a loudspeaker. On approaching a magnetic foreign body the increased inductance is converted to an audible high pitched monotone; conversely the reduced inductance caused by a nonmagnetic foreign body is converted to a bleeping sound. Thus magnetic and nonmagnetic foreign bodies are readily distinguished.

Two types of searching probes have been designed. The $T$ probe is by far the more sensitive and its main use is in the detection of minute metallic fragments some of which may have escaped radiological detection or been almost entirely altered by the process of siderosis. The spatula probe has the greatest clinical application and is used both for discrimination, when required, and for localisation at the time of surgical extraction of the foreign body. The instrument is designed to have an effectively limited range, which allows both localisation and freedom from interference by neighbouring metallic structures. The accuracy of localisation is increased by reducing the sensitivity, with a consequent further reduction in range, allowing effective localisation down to zero distance from the foreign body.

Reliability and simplicity of use are the principal attributes of the new apparatus over its predecessor.
The storage and operative temperature or humidity do not adversely influence the instrument's sensitivity, so that a high level of operative reliability is attained. Rebalancing the instrument is effected electronically by simply pressing a reset button. The apparatus is powered by a rechargeable battery, which maintains a good reserve of charge. To comply with electrical safety standards the instrument should not be used with direct mains power.

\section{Operative techniques and localisation}

With the operating microscope the sclera was exposed through a limbal conjunctival incision, and visibility was improved by muscle traction sutures. Anteroposterior and transverse movements in a serial manner were made on the sclera with the spatula probe (Fig. 2a), initially with the sensitivity raised, but then reduced, until one was satisfied that the foreign body was sited directly beneath the central aperture in the ring head of the probe. This site was then marked. In this series a full-thickness scleral trap approximately $4 \times 4 \mathrm{~mm}$ was made (it can be modified according to the expected size of the magnetic foreign body) with a razor blade fragment, and hinged posteriorly to facilitate microscopic visualisation (Fig. 2b). The underlying choroid was cauterised with the hand cautery. Two virgin silk sutures were inserted in the trap. As magnetic force varies approximately with the cube of the distance of the particle to be attracted, powerful magnets are necessary to exert traction from a distance, but a relatively weak instrument is sufficiently effective at close range. The hand-held magnet allows greater local control with continuous microscopic visualisation. Sometimes the giant magnet (Mellinger by choice) was required in cases 

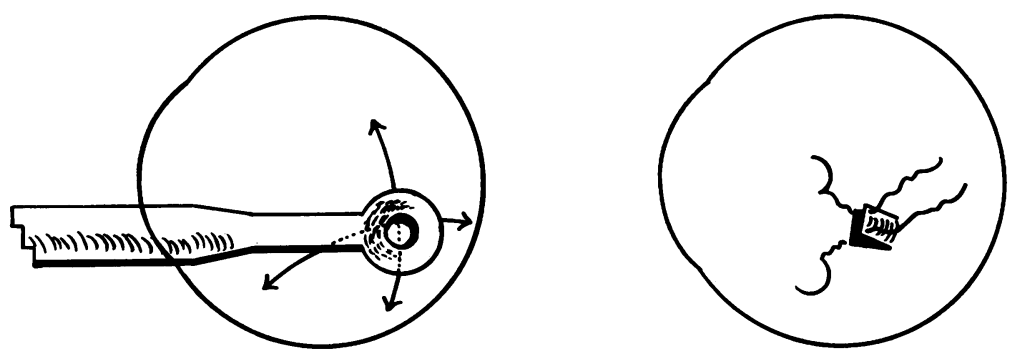

Fig. 2 (a) Searching movements with spatula probe. (b) Posteriorly hinged scleral flap

of longer standing when a fibrotic reaction might have encapsulated the foreign body.

The extraction was followed by a local vitrectomy at the extraction site and cleansing of the wound margins with hyaluronidase-soaked microsponges. The scleral trap was then repositioned, and additional sutures were inserted to effect sound closure. Cryotherapy was not routinely applied because the combined influence of the foreign body and its operative extraction have been found to exert an adequate reaction locally.

Most foreign bodies can be removed with the technique described. The difficult ones are those which are impacted in the region of the posterior pole. If discrimination confirms a nonmagnetic foreign body, the approach is different and generally necessitates an immediate removal, with vitrectomy.

\section{Results}

In 18 cases in which the preproduction model was used it successfully localised 17 foreign bodies within the posterior segment at surgery, and all were extracted as described. In the other case the foreign body was found to have passed through the posterior sclera.

Five of the patients were transferred from other ophthalmic departments; in 3 cases they were referred to this centre because of inadequate local operative facilities. Two patients were referred because of failure to extract the foreign body. The initial failed attempt at surgical removal appears to have been attributable to innaccurate localisation. In both cases accurate electroacoustic localisation led to extraction of the foreign body by the method described.

In this series 7 patients required lens surgery. The overall visual acuity was good in 14 cases. Nine patients retained a visual acuity of $6 / 6$ or better, and an additional 3 patients recovered to $6 / 9$ vision. Arrested lens opacities in 2 patients resulted in a visual acuity of $6 / 18$ in each eye. In 4 patients the visual result was $6 / 60$; the poor visual outcome in 3 of these eyes was due to a combination of macular trauma and extensive vitreous haemorrhage; the presence of siderosis in a long-standing intraocular foreign body resulted in poor vision in 1 other patient.

ADDENDUM. In the production instrument some changes and additional features have been incorporated. A foot-operated reset switch plus an additional mobile meter which can be conveniently situated for the surgeon relieve him of the need to depend on an assistant during the foreign body localisation on the operating table. A pencil probe with a similar range and sensitivity has supplanted the $\mathbf{T}$ probe. An extra large probe with a longer range is included; it allows the apparatus to be used in surgical operations on other parts of the body. The spatula probe remains the mainstay of operative ophthalmic localisation. The spatula probe alters the tone signal by a full octave in accordance with whether the foreign body lies in front of or behind the approaching probe. This facility can prove helpful in localising a metallic fragment in a large muscle mass or body cavity. In the localisation of ophthalmic foreign bodies it can be especially helpful in positions of limited exposure in establishing whether a foreign body lies impacted within the sclera and eye or is in the orbital tissues. The rechargeable battery in the production model has a longer charge, which provides about 18 hours of use.

I thank the consultant surgeons at the Birmingham and Midland Eye Hospital for permission to study their patients. My thanks are also due to Mr R. Hildred for photographic assistance and to $\mathrm{Mr} \mathrm{W}$. R. Hollingsworth for his advice on the technical descriptions.

\section{Reference}

Roper-Hall, M. J. (1954). Review of 555 Cases of intraocular foreign bodies with special reference to prognosis. British Journal of Ophthalmology, 38, 82-86. 